\title{
The Relationship between Serum Sclerostin Levels and Carotid Intima Media Thickness in Predialysis Chronic Kidney Disease Recep Demirci*1, Can Sevinc ${ }^{2}$ \\ ${ }^{1}$ Department of Nephrology, Kanuni Sultan Süleyman Research and Training Hospital, Istanbul, Turkey ${ }^{2}$ Department of Internal Medicine and Nephrology, Ataturk University Faculty of Medicine, Erzurum, Turkey *Corresponding author: Recep Demirci, E-Mail: rcpdemirci@windowslive.com, ORCID: 0000-0001-5609-9634
}

\begin{abstract}
Background: The most common cause of increased morbidity and death throughout the chronic kidney disease (CKD) process is atherosclerosis, which advances quickly and is linked to cardiovascular diseases (CVDs).

Sclerostin levels in CKD and CVDs have a complicated connection.

Objective: We examined the relationship between serum sclerostin levels and atherosclerosis in patients with stage 3,4 , or 5 CKD who were not receiving hemodialysis treatment.

Patients and Methods: A total of 125 patients aged $>18$ years with CKD stage 3, stage 4 and non-dialysis stage 5 were enrolled in the study. Patients with obesity, uncontrolled hypertension, coronary artery disease, with active cancer or liver disease, malignant hematologic disorders, acute renal failure, acute or chronic infections, were excluded. As for the control group; healthy volunteers without any known illness, regular drug use, smoking, alcohol use, and obesity were recruited.

Results: Study population was diagnosed as $39.2 \%$ with diabetes mellitus and $91.2 \%$ of patients with arterial hypertension. $54 \%$ of the patients were female. There was a positive correlation between serum sclerostin levels and carotid intima media thickness (CIMT). A negative correlation was found between serum sclerostin levels and GFR and 25-OH vitamin D levels. Sclerostin levels were found to be significantly higher in the group with high CIMT levels. There was no difference in terms of other parameters.

Conclusion: Sclerostin levels were significantly higher in patients with abnormal CIMT independent of GFR, calcium, phosphorus and PTH values. This finding suggests that the serum sclerotin level can be used as a CVD marker in patients with CKD.
\end{abstract}

Keywords: Carotid Intima Media Thickness, Predialysis Chronic Kidney Disease, Serum Sclerostin Levels.

\section{INTRODUCTION}

Chronic kidney disease (CKD) has become a serious public health concern with a global prevalence. CKD has a negative influence on one's quality of life and comes at a significant financial expense. Atherosclerosis, which progresses swiftly and is connected to cardiovascular diseases (CVDs), is the leading cause of increased morbidity and mortality throughout the CKD process (1). Although the extensive use of renal replacement therapy, the cardiovascular risk remains, and the vast majority of patients die before reaching end-stage renal failure or starting treatment ${ }^{(2)}$.

In addition to traditional risk factors that accelerate atherosclerosis in patients with CKD, the presence of non-traditional risk factors promotes the development of early rapid atherosclerosis in people with CKD. Calcium and phosphorus metabolic problems, oxidative stress, inflammation, activation of the reninangiotensin system, albuminuria, anemia, malnutrition, extracellular volume overload, and disturbance of nitric oxide and endothelial balance are the key factors that accelerate atherosclerosis ${ }^{(3)}$.

Abnormal blood phosphate levels, calcium phosphate products, and parathyroid hormone levels have all been associated to mortality in CKD patients. Vascular calcification occurs 10-20 years earlier in these individuals than in the general population ${ }^{(4)}$. Medial and intimal calcification of the arteries appears as a sign of accelerated atherosclerosis in uremic patients. Coronary artery lesions in young adults first appear as calcium crystals in the lipid core of atherosclerotic plaques ${ }^{(5)}$.

Vascular calcification was originally thought to be a passive, degenerative process that occurred in the vascular intima and media layer, but it has recently been shown to be an active, planned process ${ }^{\left({ }^{6}\right.}$. Mineral diseases such as hyperphosphatemia and hypercalcemia promote gradual calcification, which results in passive precipitation and the conversion of vascular smooth muscle cells (VSMCs) to an osteoblast-like phenotype ${ }^{(7)}$. Several proteins that are useful in this process have been discovered. The Wnt$\beta$-catenin pathway is critical for the development of skeletal and bone mass. Furthermore, this route is involved in the progression of arterial calcification and atherosclerosis ${ }^{(8-10)}$.

Sclerostin is a 22-kDa molecule and a SOST gene product. Sclerostin is released from osteocytes, inhibits the Wnt- $\beta$-catenin pathway. Sclerostin also inhibits bone formation by suppressing differentiation and proliferation of osteoblasts and stimulating osteoblast apoptosis ${ }^{(11)}$. Studies have shown that 
sclerostin levels are higher in CKD patients than in the normal population and increase as the disease progresses. However, the relationship between sclerostin levels in CKD and CVDs is inconsistent ${ }^{(\mathbf{8},}$ 12-14)

In this study, we examined the relationship between serum sclerostin levels and atherosclerosis in patients with stage 3,4 , or 5 CKD who were not receiving hemodialysis treatment.

\section{PATIENTS AND METHODS}

This study included 125 individuals above the age of 18 who had stage 3 , stage 4 , or non-dialysis stage 5 CKD. From September to December 2020, a casecontrol study was undertaken at the Health Sciences University Istanbul Kanuni Sultan Suleyman Training and Research Hospital.

\section{Ethical approval:}

The local Ethics Committee of Kanuni Sultan Süleyman Research and Training Hospital accepted this study protocol, which complies with the Declaration of Helsinki. Before beginning the study, all participants signed a written informed consent form.

According to the National Kidney Foundation K/DOQI Guidelines, all of the participants in the study had previously been diagnosed with CKD ${ }^{(15)}$. Obese individuals were excluded, as were those with uncontrolled hypertension, coronary artery disease (CAD), active malignancy or liver disease, malignant hematological illnesses, acute renal failure, or acute or chronic infections. The control group consisted of nonobese healthy volunteers $(n=22)$ with no known illnesses, habitual drug use, smoking history, or alcohol usage.

\section{Laboratory data:}

After an overnight fast, blood samples were obtained. Serum blood urea nitrogen (BUN), creatinine, sodium, potassium, calcium, phosphate, magnesium, uric acid, albumin, hemoglobin, total cholesterol, triglycerides, high-density lipoprotein, low-density lipoprotein, 25-hydroxy vitamin D, and parathyroid hormone $(\mathrm{PTH})$ were among the biochemical parameters examined.

Serum samples were frozen at $80^{\circ} \mathrm{C}$ until enzymelinked immunosorbent assays (ELISAs) were performed to evaluate sclerostin levels. The tests were performed using the BioTek ELx50 Washer and BioTek ELx800 Absorbance Reader (BioTek Instruments Inc., Winooski, VT, USA) according to the manufacturer's instructions using ELISA kits (Elabscience, Wuhan, PRC). The CKD-EPI formula was used to calculate the patients' eGFRs.

\section{Carotid intima media thickness measurement:}

The anterior neck was totally exposed with the head back in a supine position for all patients. The left and right common carotid arteries were measured separately (1 $\mathrm{cm}$ proximal to the bulbus), and the average of the two measurements was utilized. There were no measurements made in areas where atheroma plaques were visible. The intima media thickness (CIMT) of the carotid artery was measured using two echogenic lines that ran between the intima-lumen interface and the media-adventitia interface. The total of the right and left CIMTs was divided by two to get the mean CIMT. CIMT measurements were taken using the B mode of a Mindray DC-7 equipment (China) at the radiology clinic. The atherosclerosis cutoff value was $0.90 \mathrm{~mm}^{(16)}$.

\section{Statistical analysis}

IBM SPSS Statistics for Windows version 22.0 was used to conduct statistical analysis (IBM Corp., Armonk, NY, USA). To determine if the data were normally distributed, visual (histograms and probability plots) and analytical; KolmogorovSmirnov/Shapiro-tests and Wilk's methods were used. The mean and standard deviation were used to present data having a normal distribution and were compared by independent t-test.

The median (interquartile range [IQR]) were used to represent data with a non-normal distribution and were compared by Mann-Whitney $U$ test. For categorical variables, Pearson's chi-square test was utilized for comparison. The correlations between variables were discovered using correlation analysis. The Pearson's correlation coefficient was used to determine the correlation between variables using correctly dispersed data. Spearman's correlation coefficient was employed for variables with nonnormally distributed data. Significant was defined as a p-value of less than 0.05 .

\section{RESULTS}

Demographic and clinical characteristics of the study population:

In our study population, $39.2 \%$ was diagnosed with diabetes mellitus (DM) and $91.2 \%$ with arterial hypertension. The demographic and clinical characteristics of the patients are summarized in table 1 . 
Table (1): Patient demographic characteristics and laboratory values

\begin{tabular}{|c|c|}
\hline Parameter & $\begin{array}{c}\text { Mean } \pm \text { SD*/ } \\
\text { Median (IQR) }\end{array}$ \\
\hline Age (years) & $60.2 \pm 13.7$ \\
\hline $\operatorname{Sex}(n, \%)$ & \\
\hline Female & $67(53.6)$ \\
\hline Male & $58(46.4)$ \\
\hline Diabetes mellitus (n, \%) & $49(39.2)$ \\
\hline Hypertension (n, \%) & $114(91.2)$ \\
\hline eGFR $\left(\mathrm{ml} / \mathrm{dk} / 1.73 \mathrm{~m}^{2}\right)$ & $25.7 \pm 3.4^{*}$ \\
\hline BUN (mg/dl) & $43 \pm 9^{*}$ \\
\hline Creatinine (mg/dl) & $2.32(1.76-3.4)^{\#}$ \\
\hline Calcium (mg/dl) & $9.1 \pm 0.08^{*}$ \\
\hline Phosphate (mg/dl) & $3.9 \pm 0.1^{*}$ \\
\hline Magnesium (mg/dl) & $1.95 \pm 0.3^{*}$ \\
\hline Uric acid (mg/dl) & $6.4 \pm 1.5^{*}$ \\
\hline Albumin (gr/dl) & $4.1(3.8-4.4)^{\#}$ \\
\hline Hemoglobin (g/dl) & $11.5 \pm 2.2^{*}$ \\
\hline Total cholesterol (mg/dl) & $187.5 \pm 8.9^{*}$ \\
\hline Triglycerides (mg/dl) & $147.5(103.8-221.3)^{\#}$ \\
\hline HDL (mg/dl) & $43.2 \pm 11.3^{*}$ \\
\hline LDL (mg/dl) & $109 \pm 3.9 *$ \\
\hline 25-OH vitamin $\mathrm{D}(\mathrm{ng} / \mathrm{ml})$ & $16.5(11.2-24.9)^{\#}$ \\
\hline PTH $(\mathrm{pg} / \mathrm{ml})$ & $98(68-215.5)^{\#}$ \\
\hline Mean CIMT (mm) & $0.68 \pm 0.19 *$ \\
\hline Sclerostin (pg/ml) & $171.5(145.2-222.4)^{\#}$ \\
\hline
\end{tabular}

IQR: interquartile range, eGFR: estimated glomerular filtration rate, BUN: blood urea nitrogen, HDL: high-density lipoprotein, LDL: low-density lipoprotein, PTH: parathyroid hormone, CIMT: carotid intima media thickness

No significant difference in sclerostin level was observed between the sexes $(\mathrm{p}=0.331)$. The mean CIMT of the patients was $0.68 \pm 0.9 \mathrm{~mm}$, and the mean CIMT of the control group was $0.41 \pm 0.03 \mathrm{~mm}(\mathrm{p}<0.001)$. A significant difference in sclerostin level was observed between participants with and without DM (171.7, IQR: 161.8-248.9 vs. 169.1, IQR: $136.7-200.9 ; \mathrm{p}=0.022)$, but there was no difference between patients with and without hypertension ( $\mathrm{p}$ $=0.383$ ). Significant differences in the serum sclerostin level were observed between stage 3 and stage 4 CKD patients $(\mathrm{p}<0.001)$ and between stage 3 and stage 5 CKD patients $(\mathrm{p}=0.05)$ (Figure 1$)$.
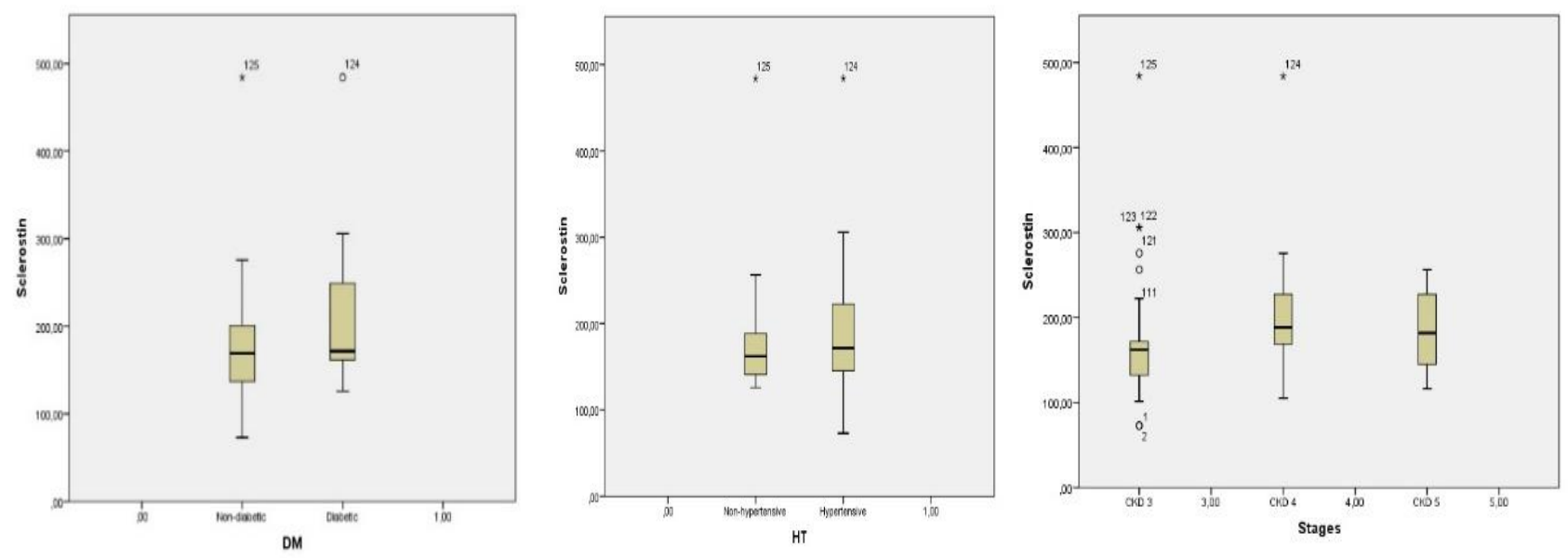

Figure (1): Serum sclerostin levels according to diabetes mellitus (DM) status, hypertension (HT) status, and chronic kidney disease stage. 
Relationships between serum sclerostin level and other parameters:

The presence of DM, BUN, PTH, and CIMT was found to have a positive connection with serum sclerostin levels. The eGFR and the 25-OH vitamin D level were shown to have a negative relationship (Table 2).

Table (2): Results of correlation analysis between biochemical parameters and serum sclerostin levels

\begin{tabular}{|c|c|c|}
\hline Parameter & $r$ & $p$-value \\
\hline Age (years) & 0.140 & 0.121 \\
\hline DM & 0.206 & 0.021 \\
\hline HT & 0.078 & 0.385 \\
\hline eGFR $\left(\mathrm{ml} / \mathrm{dk} / 1.73 \mathrm{~m}^{2}\right)$ & -0.181 & 0.043 \\
\hline BUN (mg/dl) & 0.189 & 0.035 \\
\hline Creatinine (mg/dl) & 0.029 & 0.751 \\
\hline PTH (pg/ml) & 0.184 & 0.040 \\
\hline Right CIMT (mm) & 0.326 & $<0.001$ \\
\hline Left CIMT (mm) & 0.338 & $<0.001$ \\
\hline Mean CIMT (mm) & 0.343 & $<0.001$ \\
\hline Phosphate (mg/dl) & 0.119 & 0.186 \\
\hline Calcium (mg/dl) & -0.061 & 0.497 \\
\hline $25-\mathrm{OH}$ vitamin D (ng/ml) & -0.237 & 0.008 \\
\hline
\end{tabular}

R: correlation coefficient, DM: diabetes mellitus, HT: hypertension, eGFR: estimated glomerular filtration rate, BUN: blood urea nitrogen, CIMT: carotid intima media thickness

\section{Comparison between high- and low-sclerostin groups:}

As in earlier investigations, the patients $(n=125)$ were separated into high- and low-sclerostin groups based on the median level $(171.5 \mathrm{pg} / \mathrm{ml})$. The high-sclerostin group had significantly higher mean age, BUN, creatinine, phosphate, PTH, and CIMT than the low-sclerostin group. The high-sclerostin group had significantly lower eGFR, calcium levels, and 25-OH vitamin D levels than the low-sclerostin group (Table 3).

Table (3): Comparisons between high- and low-sclerostin groups

\begin{tabular}{|c|c|c|c|}
\hline Parameter & $\begin{array}{c}\text { High-sclerostin group } \\
(\mathbf{n}=60) \\
\text { Mean } \pm \text { SD } * / \\
\text { Median (IQR) })^{\#}\end{array}$ & $\begin{array}{c}\text { Low-sclerostin group } \\
(\mathbf{n}=65) \\
\text { Mean } \pm \text { SD } * / \\
\text { Median }(\text { IQR })^{\#}\end{array}$ & $p$-value \\
\hline Age (years) & $63.2 \pm 2.4^{*}$ & $57.4 \pm 4.3^{*}$ & 0.017 \\
\hline eGFR $\left(\mathrm{ml} / \mathrm{dk} / 1.73 \mathrm{~m}^{2}\right)$ & $21.4 \pm 1.4^{*}$ & $29.7 \pm 4.7 *$ & 0.001 \\
\hline BUN (mg/dl) & $48.3 \pm 8.4^{*}$ & $38.2 \pm 8.4^{*}$ & $<0.001$ \\
\hline Creatinine (mg/dl) & $2.6(1.9-4)^{\#}$ & $2.1(1.6-3.4)^{\#}$ & $<0.001$ \\
\hline Calcium (mg/dl) & $9 \pm 0.8^{*}$ & $9.3 \pm 0.7 *$ & 0.027 \\
\hline Phosphate (mg/dl) & $4 \pm 0.9^{*}$ & $3.9 \pm 0.3^{*}$ & 0.049 \\
\hline $25-\mathrm{OH}$ vitamin $\mathrm{D}(\mathrm{ng} / \mathrm{ml})$ & $14.3(10-20.4)^{\#}$ & $18.5(12.1-27.2)^{\#}$ & 0.034 \\
\hline PTH (pg/ml) & $205(88.3-217.8)^{\#}$ & $93(54-235.5)^{\#}$ & $\mathbf{0 . 0 3 7}$ \\
\hline Right CIMT (mm) & $0.69 \pm 0.18^{*}$ & $0.63 \pm 0.16^{*}$ & $\mathbf{0 . 0 3 2}$ \\
\hline Left CIMT (mm) & $0.75 \pm 0.12^{*}$ & $0.67 \pm 0.10^{*}$ & 0.021 \\
\hline Mean CIMT (mm) & $0.72 \pm 0.19^{*}$ & $0.65 \pm 0.17^{*}$ & 0.021 \\
\hline
\end{tabular}

DM: diabetes mellitus, HT: hypertension, eGFR: estimated glomerular filtration rate, BUN: blood urea nitrogen, CIMT: carotid intima media thickness.

\section{Serum sclerostin levels and CIMT:}

Patients were separated into two groups based on whether their CIMT was greater than $0.90 \mathrm{~mm}$ or less than $0.90 \mathrm{~mm}$. The group with a higher CIMT had considerably higher sclerostin levels. There were no variations in the other characteristics (Table 4). 
Table (4): Comparison of the patients according to CIMT

\begin{tabular}{|c|c|c|c|}
\hline Parameter & $\begin{array}{c}\text { CIMT }<0.90 \mathrm{~mm} \\
(\mathrm{n}=87)\end{array}$ & $\begin{array}{c}\text { CIMT } \geq 0.90 \mathrm{~mm} \\
(\mathrm{n}=38)\end{array}$ & $p$-value \\
\hline Sclerostin (pg/ml) & $168.9(145.2-196.6)^{\#}$ & $216.7(169.1-275.7)^{\#}$ & $<0.001$ \\
\hline Age (years) & $58.5 \pm 4.7 *$ & $64.1 \pm 10.3^{*}$ & 0.057 \\
\hline $\operatorname{eGFR}\left(\mathrm{ml} / \mathrm{dk} / 1.73 \mathrm{~m}^{2}\right)$ & $25 \pm 4.3 *$ & $27.4 \pm 1.3^{*}$ & 0.284 \\
\hline BUN (mg/dl) & $42.7 \pm 2.1^{*}$ & $43.8 \pm 3.1^{*}$ & 0.186 \\
\hline Creatinine (mg/dl) & $2.4(1.7-4.2)^{\#}$ & $2.3(1.8-3.1) \#$ & 0.261 \\
\hline Calcium (mg/dl) & $9.1 \pm 0.8^{*}$ & $9.4 \pm 0.7^{*}$ & 0.305 \\
\hline Phosphate (mg/dl) & $4 \pm 1.3^{*}$ & $3.7 \pm 0.7 *$ & 0.057 \\
\hline $25-\mathrm{OH}$ vitamin D $(\mathrm{ng} / \mathrm{ml})$ & $16.7(11.8-25.4)^{\#}$ & $14.4(9.5-23.6)^{\#}$ & 0.221 \\
\hline PTH (pg/ml) & $101(63-292)^{\#}$ & $98(90-147) \#$ & 0.601 \\
\hline
\end{tabular}

CIMT: carotid intima media thickness, eGFR: estimated glomerular filtration rate, BUN: blood urea nitrogen.

\section{Serum sclerostin cut-off value for predicting atherosclerosis:}

Receiver operating characteristic curve analysis was performed to evaluate the usefulness of sclerostin as a marker for atherosclerosis. The area under the curve was $0.688(\mathrm{p}=0.001,95 \%$ CI $0.57-0.800)$ for an abnormal CIMT thickness. A concentration > 170.9 $\mathrm{pg} / \mathrm{ml}$ was associated with a sensitivity of $76.3 \%$ and specificity of $58.6 \%$ in terms of identifying an increased risk of an abnormal CIMT.

\section{DISCUSSION}

Patients with CKD had greater sclerostin levels than the general population, and the levels increased as the disease advanced, according to our findings. The findings demonstrate that serum sclerostin levels are negatively associated to renal function in individuals with stage 3-5 CKD, implying that sclerostin could be a useful biomarker in the disease. Despite the fact that serum sclerostin levels in individuals with CKD are high, the basic mechanisms that generate these high levels have yet to be fully understood. In a mouse genetic model of CKD, Sabbagh et al. $^{\left({ }^{(17)}\right.}$ found that inhibited osteocyte Wnt - catenin signaling and elevated production of sclerostin occurred during the early stages of the disease. According to Cejka et al. ${ }^{(\mathbf{1 8})}$ reduced eGFR increased urinary sclerostin excretion, however greater sclerostin levels were not related with poorer renal function in CKD patients. Graciolli et al. ${ }^{\left({ }^{(9)}\right)}$ suggested that the percentage of sclerostin-positive osteocytes is high in patients with CKD and that high levels of sclerostin are likely related to this high percentage. Sclerostin is mostly formed from osteocytes, according to immunohistochemistry, and increased serum sclerostin may be obtained in part from calcified vasculature ${ }^{(20)}$. A relationship between the serum sclerostin level and sex was detected in previous studies, with male patients having higher sclerostin levels. This has been attributed to the high bone mass and low estrogen levels in men, as estrogens inhibit sclerostin ${ }^{(\mathbf{8}, 21)}$. In our study, no relationship was found between sex and sclerostin level, possibly because of the small number of patients. However, as in previous studies, we found a relationship between age and sclerostin (21, 22); the ages of patients with high sclerostin levels were significantly higher.

Patients with CKD are at an increased risk of premature mortality, mainly from cardiovascular causes. Relationship between CKD and hemodialysis and atherosclerosis has been known. It has been suggested that there is an increased risk of atherosclerosis during the early stages of CKD, with the risk level remaining constant thereafter ${ }^{(23)}$.

Atherosclerosis is a multifaceted condition that includes vascular calcification, inflammation, endothelial dysfunction, and VSMC proliferation and migration. Previously, vascular calcification was thought to be a passive and degenerative process that occurs in the arterial intima and media layers. However, the vascular calcification process is actively regulated by many interconnected unique mechanisms (6). Chronic inflammation, which plays a role in the atherosclerosis process, also regulates the mechanisms that cause vascular calcification. Recent studies have clarified the importance of angiogenesis in the development of vascular calcification and the roles of osteoblasts and osteoclasts in this event. Vascular calcification is seen in the intima layer where atherosclerosis occurs, the media layer with Mönckeberg sclerosis, and the heart valves. The mechanism underlying vascular calcification involves the activation of osteogenesis in the vascular wall, deficiency of factors preventing calcification, increased bone turnover, and disorders in mineral metabolism ${ }^{(24)}$.

Many cells in the vascular wall transform into osteogenic and chondrogenic cells and play roles in the vascular calcification process. VSMCs and adventitial pericyte cells are most susceptible to change and are the main cells involved in this process. Proteins such as osteonectin, osteopontin, PTH, bone morphogenic 
protein-2, and the matrix-Gla protein released from these cells play direct or indirect roles in atherosclerotic plaques and calcified areas. In addition, the presence of osteoblast and osteoclast-like cells in the circulation and vascular calcification areas reveals the relationship between the formation of calcification and bone metabolism. VSMCs and osteoblasts originate from common mesenchymal cells during the embryogenic period. These cells are transformed into osteoblasts in the presence of phosphate in the environment, and a mineralized matrix forms. The transcription factor RUNX2/Cfba-1 is thought to play a role in this transformation ${ }^{(25)}$.

Osteoclasts are multinuclear cells derived from mononuclear cell precursors. The factors responsible for the maturation of osteoclasts include monocyte colony-stimulating factor, receptor activator for nuclear factor-B (RANK), and RANK ligand (RANKL). These cytokines are detectable in atherosclerotic plaques and are responsible for ossification of the arterial wall. Osteoprotegerin (OPG), RANK, and RANKL in the tumor necrosis factor (TNF) family are involved in regulating both osteoclast functions and the immunomodulatory effects of $\mathrm{T}$ and $\mathrm{B}$ lymphocytes ${ }^{(26)}$. Recent studies have emphasized the importance of inflammation in the development of vascular calcification. It has been shown that inflammation plays a role in bone formation and destruction, particularly in the transformation of VSMCs into osteogenic cells, the formation of osteoblasts, and the precipitation of hydroxyapatite crystals. Many inflammatory cytokines that play a role in bone loss are also involved in osteoclastogenesis. The release of OPG and RANKL from vascular cells increases due to the effects of interleukin- 1 and TNF- $\alpha$, which are pro-inflammatory cytokines, whereas anti-inflammatory cytokines, such as TGF- $\beta$, suppress OPG production ${ }^{(24)}$. In the pathogenesis of atherosclerosis, Wnt-catenin signaling is crucial. Endothelial inflammation, mesenchymal stem cell development, and VSMC proliferation and survival are all regulated by it. Monocyte adherence to endothelial cells is aided by the Wnt protein ${ }^{(27-29)}$.

Sclerostin is thus a powerful bone formation and mineralization inhibitor ${ }^{(30)}$. In diabetic and CKD patients, studies have found a link between serum sclerostin levels and the presence of atherosclerosis and atherosclerotic plaques $(\mathbf{8 , 1 2 , 1 6 )}$. The CIMT as measured using ultrasonography is an inexpensive, easily applied, reproducible, non-invasive indicator that can be used to evaluate the presence and extent of atherosclerosis. CIMT measurements can be used to determine atherosclerotic cardiovascular disease at an early stage ${ }^{(31)}$. The carotid arterial system can be easily visualized, and it has laminar flow patterns that are affected during the development of atherosclerosis. Determining the prevalence of atherosclerosis in these arteries also provides information about the presence and severity of accompanying coronary atherosclerosis. Studies have reported that the CIMT is an indicator of atherosclerosis that is correlated with myocardial infarction, stroke, and peripheral artery disease ${ }^{(32,33)}$. Again, in studies conducted on CKD, it has been revealed that the CIMT is associated with CVDs according to ultrasonographic studies of patients with CKD ${ }^{(34,35)}$. The level of sclerostin and CIMT were found to have a positive connection in our investigation. Patients with high sclerostin levels had high CIMT results. Patients with a CIMT $>0.90 \mathrm{~mm}$ had significantly greater sclerostin levels.

The small number of participants in our study and the exclusion of patients with CAD are the study's limitations. Furthermore, it should be proven on a wide scale whether sclerostin levels are independently linked with cardiovascular events and survival in individuals with atherosclerotic disease.

\section{CONCLUSION}

Sclerostin levels were considerably greater in patients with an abnormal CIMT, regardless of eGFR, calcium, phosphate, or PTH levels, according to our findings. This study implies that the level of serum sclerotin in CKD patients can be used as a CVD marker.

\section{REFERENCES}

1. Tonelli M, Wiebe $\mathrm{N}$, Culleton B et al. (2006): Chronic kidney disease and mortality risk: a systematic review. J Am Soc Nephrol., 17(7):2034-47.

2. Slinin Y, Greer N, Ishani A et al. (2015): Timing of dialysis initiation, duration and frequency of hemodialysis sessions, and membrane flux: a systematic review for a KDOQI clinical practice guideline. Am J Kidney Dis., 66(5):823-36.

3. Sarnak M, Coronado B, Greene T et al. (2002): Cardiovascular disease risk factors in chronic renal insufficiency. Clin Nephrol., 57(5):327-35.

4. Braun J, Oldendorf M, Moshage W et al. (1996): Electron beam computed tomography in the evaluation of cardiac calcification in chronic dialysis patients. Am J Kidney Dis., 27(3):394-401.

5. Stary H (1990): The sequence of cell and matrix changes in atherosclerotic lesions of coronary arteries in the first forty years of life. Eur Heart J., 11:3-19.

6. Jono S, Shioi A, Ikari Y et al. (2006): Vascular calcification in chronic kidney disease. J Bone Miner Metab., 24(2):176-81.

7. Ketteler M, Westenfeld R, Schlieper G et al. (2005): Pathogenesis of vascular calcification in dialysis patients. Clin Exp Nephrol., 9(4):265-270.

8. Chen A, Sun Y, Cui J et al. (2018): Associations of sclerostin with carotid artery atherosclerosis and allcause mortality in Chinese patients undergoing maintenance hemodialysis. BMC Nephrol., 19(1):264269.

9. Johnson M, Rajamannan N (2006): Diseases of Wnt signaling. Rev Endocr Metab Disord. Jun;7(1-2):41-9. Erratum in: Rev Endocr Metab Disord., 8(2):183. 
10. Evrard S, Delanaye P, Kamel S et al. (2015): Vascular calcification: from pathophysiology to biomarkers. Clin Chim Acta., 438:401-14.

11. Zeng C, Guo C, Cai J et al. (2018): Serum sclerostin in vascular calcification and clinical outcome in chronic kidney disease. Diab Vasc Dis Res., 15(2):99105.

12. Figurek A, Spasovski G (2018): Is serum sclerostin a marker of atherosclerosis in patients with chronic kidney disease-mineral and bone disorder? Int Urol Nephrol., 50(10):1863-1870.

13. Stavrinou E, Sarafidis P, Koumaras C et al. (2019): Increased sclerostin, but not Dickkopf-1 protein, is associated with elevated pulse wave velocity in hemodialysis subjects. Kidney Blood Press Res., 44(4):679-689.

14. Qureshi A, Olauson H, Witasp A et al. (2015): Increased circulating sclerostin levels in end-stage renal disease predict biopsy-verified vascular medial calcification and coronary artery calcification. Kidney Int., 88(6):1356-1364.

15. KDIGO CKD Work Group (2013): KDIGO 2012 clinical practice guideline for the evaluation and management of chronic kidney disease. Kidney Int Suppl., 3: 1-150.

16. Shalash M, Rohoma $K$, Kandil $N$ et al. (2019): Serum sclerostin level and its relation to subclinical atherosclerosis in subjects with type 2 diabetes. J Diabetes Complications, 33(8):592-597.

17. Sabbagh Y, Graciolli F, O'Brien $S$ et al. (2012): Repression of osteocyte $\mathrm{Wnt} / \beta$-catenin signaling is an early event in the progression of renal osteodystrophy. Journal of Bone and Mineral Research, 27: 1757-1772.

18. Cejka D, Marculescu R, Kozakowski N et al. (2014): Renal elimination of sclerostin increases with declining kidney function. J Clin Endocrinol Metab., 99(1):248-55.

19. Graciolli F, Neves K, Barreto F et al. (2017): The complexity of chronic kidney disease-mineral and bone disorder across stages of chronic kidney disease. Kidney Int., 91:1436-1446.

20. Brandenburg V, Kramann R, Koos R et al. (2013): Relationship between sclerostin and cardiovascular calcification in hemodialysis patients: a cross-sectional study. BMC Nephrol., 14:219.

21. Modder U, Hoey K, Amin S et al. (2011): Relation of age, gender, and bone mass to circulating sclerostin levels in women and men. J Bone Miner Res., 26:3739.

22. Viaene L, Behets G, Claes K et al. (2013): Sclerostin: another bone-related protein related to all-cause mortality in haemodialysis? Nephrol Dial Transplant., 28:3024-30.

23. Valdivielso J, Rodríguez-Puyol D, Pascual J et al. (2019): Atherosclerosis in chronic kidney disease: more, less, or just different? Arterioscler Thromb Vasc Biol., 39(10):1938-1966.

24. Derici U, Nahas A (2006): Vascular calcification in uremia: Old concepts and new insights. Semin Dial., 19(1):60-68.

25. Hruska K, Mathew S, Saab G (2005): Bone morphogenetic proteins in vascular calcification. Circ Res., 97(2):105-14.

26. Kazama J (2004): Osteoprotegerin and bone mineral metabolism in renal failure. Current Opinion in Nephrology and Hypertension, 13:411-415.

27. Kim J, Kim J, Kim D et al. (2010): Wnt5a induces endothelial inflammation via beta-catenin-independent signaling. J Immunol., 185:1274-1282.

28. Lee $D$, Nathan Grantham $R$, Trachte A et al. (2006): Activation of the canonical Wnt/beta catenin pathway enhances monocyte adhesion to endothelial cells. Biochem Biophys Res Commun., 347:109-116.

29. Mill C, George S (2012): Wnt signaling in smooth muscle cells and its role in cardiovascular disorders. Cardiovasc Res., 95:233-240.

30. Brandenburg V, D'Haese P, Deck A et al. (2016): From skeletal to cardiovascular disease in 12 steps-the evolution of sclerostin as a major player in CKDMBD. Pediatr Nephrol., 31(2):195-206.

31. Salonen J, Salonen R (1991): Ultrasonographically assessed carotid morphology and risk of coronary heart disease. Arterioscler Thromb., 11:1245-9.

32. O'Leary D, Polak J, Kronmal R et al. (1999): Carotid-artery intima and media thickness as a risk factor for myocardial infarction and stroke in older adults: Cardiovascular Health Study Collaborative Research Group. N Engl J Med., 340:14-22.

33. Benedetto FA, Mallamaci F, Tripei G, Zocalli C (2001): Prognostic value of ultrasonographic measurement of carotid intima media thickness in dialysis patients. J Am Soc Neephrol., 12:2458-2464.

34. Szeto C, Chow K, Woo K et al. (2007): Carotid intima media thickness predicts cardiovascular disease in Chinese patients with chronic kidney disease. J Am Soc Neephrol., 18:1966-1972.

35. Betriu-Bars Á, Fernández-Giráldez E (2012): Carotid ultrasound for the early diagnosis of atherosclerosis in chronic kidney disease. Nefrologia, 32(1):7-11. 